\title{
CHAOS AND MUSIC: FROM TIME SERIES ANALYSIS TO EVOLUTIONARY COMPOSITION
}

\author{
MAXIMOS A. KALIAKATSOS-PAPAKOSTAS \\ Department of Mathematics, University of Patras, \\ GR-26110 Patras, Greece \\ maxk@math.upatras.gr \\ MICHAEL G. EPITROPAKIS \\ Computing Science and Mathematics, \\ School of Natural Sciences, University of Stirling, \\ Stirling FK9 4LA, Scotland, UK \\ mge@cs.stir.ac.uk \\ ANDREAS FLOROS \\ Department of Audio and Visual Arts, Ionian University, \\ GR-49100 Corfu, Greece \\ floros@ionio.gr \\ MICHAEL N. VRAHATIS \\ Department of Mathematics, University of Patras, \\ GR-26110 Patras, Greece \\ vrahatis@math.upatras.gr
}

Received February 7, 2013; Revised July 16, 2013

\begin{abstract}
Music is an amalgam of logic and emotion, order and dissonance, along with many combinations of contradicting notions which allude to deterministic chaos. Therefore, it comes as no surprise that several research works have examined the utilization of dynamical systems for symbolic music composition. The main motivation of the paper at hand is the analysis of the tonal composition potentialities of several discrete dynamical systems, in comparison to genuine human compositions. Therefore, a set of human musical compositions is utilized to provide "compositional guidelines" to several dynamical systems, the parameters of which are properly adjusted through evolutionary computation. This procedure exposes the extent to which a system is capable of composing tonal sequences that resemble human composition. In parallel, a time series analysis on the genuine compositions is performed, which firstly provides an overview of their dynamical characteristics and secondly, allows a comparative analysis with the dynamics of the artificial compositions. The results expose the tonal composition capabilities of the examined iterative maps, providing specific references to the tonal characteristics that they can capture.
\end{abstract}

Keywords: Chaos and music; tonal time series; evolutionary composition.

\section{Introduction}

Music composition is a process that encompasses a combination of two contradicting forces: the determinism imposed by music rules and the chaos that is subsumed in human creativity. Therefore, the utilization of chaotic system for automatic music composition seems like a direction worthy of extensive exploration. In fact, several research works have focused on the potential of using dynamical systems for music composition, however, the 
compositional potentiality of dynamical systems remains relatively unexplored. Although many methodologies have been proposed, the results that most works have presented do not provide comparative analyses between the dynamical systems' compositions and the ones composed by humans. As a result, one may not come up with practical implications as to whether a human-like "chaotic composer" can be created. If so, what would its dynamical and musical characteristics be? The aims of this paper may be summarized as the examination of symbolic music compositional capabilities of several dynamical systems, focusing on the tonal aspects of music.

Nonlinear characteristics are deeply related to the human perception of sound. Their existence in natural sound has been studied for a large variety of phenomena, such as animal vocalization [Tokuda et al., 2002] and birdsong production models [Amador \& Mindlin, 2008]. Furthermore, the relation of nonlinear dynamics with human speech has been investigated [Behrman, 1999], providing also significant results in speech recognition [Jafari et al., 2010]. Additionally, theories that incorporate nonlinearities in the mechanism through which humans perceive auditory events have provided great insights about sound and human perception [Chialvo, 2003]. Consequently, it comes without saying that the ultimate human cultural manifestation of sound - music - abounds in nonlinear structures on many levels: from the level of instrumental timbre [Fletcher, 1994] and overall orchestration timbres [Voss \& Clarke, 1978], to the level of symbolic composition [Manaris et al., 2005].

Automatic music composition through dynamical systems has been a very popular technique which inspired many researchers and artists, from the pioneering works of Pressing [1988], Bidlack [1992], Herman [1993] and Harley [1994], to more contemporary works. An interesting approach for altering the tonal characteristics of a piece was proposed in [Dabby, 1996], where the pitch space of the specific piece was "convolved" with a chaotic solution of a dynamical system. Thereafter, new neighboring solutions provided novel tonal sequences that gradually diverged from the ones of the initial piece. The mainstream compositional strategy however, is the utilization of well-known dynamical systems like Chua's circuit [Chua et al., 1993] for the generation of sound [Choi, 1994] or music composition [Bilotta et al., 2007; Rizzuti et al., 2009]. The latter works not only examine the composition of music with Chua's circuit, but also evolve its parameters with genetic algorithms. The fitness of the evolutionary process is provided by the pitch interval distribution of a target piece. Several dynamical systems have been examined in [Coca et al., 2010] and the produced music was characterized according to several music characteristics. A similar approach was followed in [KaliakatsosPapakostas et al., 2012b], but the musical output in this case was examined in terms of its information complexity.

The paper at hand is motivated by the potentialities of dynamical systems for "symbolic" composition of musical tonal sequences, reflected in numerous works that employ such systems for automatic composition. Since the focus is on the tonal aspect of music, the automatic composition methodology that is followed disregards information on rhythm, intensities and timbre. To this end, two types of analysis are applied, which incorporate the extraction of several tonal characteristics from genuine music masterpieces and the composition of novel tonal sequences using chaotic systems and evolution. On the one hand, the genuine compositions are examined in terms of their dynamical characteristics through a time series analysis approach which incorporates phase space reconstruction of the pieces' attractors, the extraction of the largest Lyapunov exponent and fractal dimension. A similar approach was presented in [Boon \& Decroly, 1995], but the present paper provides an exhaustive experimental research on a large set of compositions, combined with a comparative analysis on the time series characteristics between genuine and artificial compositions. On the other hand, several well-known dynamical systems are utilized to compose novel tonal sequences, which are "trained" to share similar characteristics with the aforementioned pieces. In the latter analysis, the parameters of these dynamical systems are optimized through evolutionary computation, so that the generated novel pieces share similar characteristics to the respective "target" genuine ones.

These tonal characteristics are exhibited to highlight the exceptional characteristics of different music styles and therefore constitute a qualitative measurement of the potentialities of each dynamical system concerning music composition. Through 
an extensive experimental study, the tonal sequences produced by some of the examined discrete dynamical systems are observed to encompass some tonal characteristics that are present in genuine human compositions. However, through the phase space reconstruction that is realized with the time series analysis of the genuine pieces, it is indicated that dynamical systems with higher complexity and with a greater number of dimensions are required to compose human-like music. These results are assessed through a comparative scrutinization between the genuine and the artificial compositions, not only on the level of musical characteristics, but also on the level of their dynamical behavior expressed with the largest Lyapunov exponent and fractal dimension.

The rest of the paper is organized as follows. Section 2 discusses the extraction of tonal time series from genuine symbolic compositions in MIDI format and presents the tools for the time series analysis, which allows the phase space reconstruction and the computation of the largest Lyapunov exponent and fractal dimension. The utilized automatic music composition methodology is reviewed in detail in Sec. 3. Specifically, the evolutionary scheme that allows the tuning of the dynamical systems' parameters is presented and the music features that constitute the basis for the fitness function are throughly analyzed. Section 3 also discusses the automatic tonal composition methodology which encompasses coarse compositional guidelines to the dynamical systems. A thorough experimental report is given in Sec. 4, which incorporates the most important findings that were extracted by the application of the methodologies described in the preceding sections. The paper concludes in Sec. 5 with a synopsis along with concluding remarks and some pointers to future work.

\section{Dynamical Properties of the Genuine Compositions Through Time Series Analysis}

As mentioned previously, this work is targeted towards studying the tonal composition potentialities of discrete dynamical systems, in the context of automatic music composition. Therefore, the output produced in consecutive iterations of these dynamical systems, is mapped to tonal sequences through a straightforward procedure that is described below in detail. An initial approach to this subject is the study of tonal sequences that derive from "genuine" music masterpieces, in terms of their dynamical characteristics. In order to consider a wide spectrum of Western music, this study incorporates several genuine compositions from J. S. Bach, Mozart, Beethoven and a collection of jazz standards composed by various artists. For the rest of the paper, the entire jazz collection will be referred to as if it were composed by a single composer for simplicity. The fact that all jazz compositions are considered per composer is not expected to affect the accuracy of results, because the jazz compositional technique varies significantly from that of classical music. Therefore, their inclusion in a single category is intended to examine the differences in dynamical properties among the compositional styles of the collected music sets. The extraction of dynamical characteristics from the tonal sequences that derive from all the aforementioned compositions allows a first cartographical overview of the underlying dynamics that are considered to produce these compositions. Furthermore, this analysis allows a comparison in the dynamical characteristics of the genuine and the artificial compositions.

The composition process that is assumed throughout the paper regards the symbolic level, i.e. only information that is interpretable in a score is considered, without any timbre-related information. Therefore, the aforementioned genuine compositions are collected in MIDI format, which is a protocol that includes the most viable symbolic information of music content, allowing a quite accurate interpretation to music score. Additionally, in order to have comparable results among the time series extracted from the genuine pieces, only piano executions were considered. Specifically, the dataset comprises piano sonatas from Beethoven and Mozart, the "Well Tempered Clavier" of Bach and piano transcriptions of several well-known jazz standards. This fact "neutralizes" the effect of instrument-imposed constraints, like tonal range and polyphony, that may affect the format of the tonal sequences. To this end, the tonal sequence of a piece is obtained by the serial concatenation of all its tones in the order they appear, disregarding inter-onset distance and duration. In the case of polyphonic events, i.e. music events that incorporate multiple simultaneous tones, the order of tones is considered from the lowest to the highest tone 
of the polyphonic cluster. Although this approach might be considered simplistic, it reflects the qualitative demeanor of this study. ${ }^{1}$

\subsection{Phase space reconstruction}

Based on Takens's [1981] theorem, if a time series, $\left\{x_{i}\right\}_{i \in\{0,1,2, \ldots, n\}}$, derives from a dynamical system, it encompasses all the viable characteristics needed to construct a topologically equivalent dynamical system. Thereby, the dynamical behavior of the initial system can be "reconstructed" in a topologically consistent manner, even if the system's dimensionality is higher than the observed time series'. This procedure is called "phase space reconstruction" and is realized by estimating the "novelty" introduced in segments of the time series (to compute a proper time delay) and then computing the dimensionality of the dynamical system's space. The phase space of the dynamical system is then reconstructed by embedding replicates of the time series, shifted by a proper index expressed by an optimal time lag, in each dimension of the reproduced system. The resulting dynamical system is considered to produce the following vector sequence, $\mathbf{y}(i)=\left(x_{i}, x_{i+\tau}, x_{i+2 \tau}, \ldots, x_{i+(\mu-1) \tau}\right)$. A brief review of this methodology is discussed in the following paragraphs, while for a more thorough review the reader is referred to [Lai \& Ye, 2003; Kodba et al., 2005]. In the paper at hand, the software implementation presented in [Kugiumtzis \& Tsimpiris, 2010] was utilized. The solicitation of further dynamical information is realized through two more measures, namely the Largest Lyapunov Exponent (LLE) and the Fractal Dimension (FD) as described later. The latter two dynamical measures are not necessary for the phase space reconstruction, they are rather used as measures of comparison between the genuine and the automatically produced compositions.

\subsubsection{Embedding delay}

The rationale behind determining a proper time delay is the allocation of a time series segmentation to equal segments of length $\tau$, where each segment incorporates information about the other. Such a segmentation exposes the interrelations that the underlying dynamical system imposes to each segment of the time series. The time delay (or segment length) value, $\tau$, should be chosen so that the variables $x_{i}, x_{i+\tau}, x_{i+2 \tau}, \ldots, x_{i+(q-1) \tau}$, where $q=$ $\lfloor l / \tau\rfloor$ and $l$ is the length of the time series, are as independent as possible. To this end, Fraser and Swinney [1986] proposed the examination of the mutual information between all segments of the time series, for various $\tau$ values. The computation of mutual information is performed with the MATS [Kugiumtzis \& Tsimpiris, 2010] toolbox in MATLAB, in which a partitioning is considered that produces a grid in the time series domain interval $\left[\min \left\{x_{i}\right\}, \max \left\{x_{i}\right\}\right]_{i \in\{0,1,2, \ldots, n\}}$, with $k$ partitions of length $k /\left(\max \left\{x_{i}\right\}-\min \left\{x_{i}\right\}\right)_{i \in\{0,1,2, \ldots, n\}}$. Given the aforementioned domain partitioning, the mutual information for a time delay $\tau$ is given by [Kodba et al., 2005]

$I(\tau)=-\frac{1}{l-\tau} \sum_{n=1}^{k} \sum_{m=1}^{k} P_{n, m}(\tau) \log \frac{P_{n, m}(\tau)(l-\tau)}{P_{n} P_{m}}$,

where $l$ is the length of the time series, $P_{n}$ and $P_{m}$ are the probabilities that a time series value is in the $n$th and $m$ th grid position respectively and $P_{n, m}(\tau)$ is the joint probability that $x_{i}$ is in the $n$th grid position and $x_{i+\tau}$ is in the $m$ th grid position. The first minimizer of $I(\tau)$ constitutes the proper time delay (segmentation length), to obtain the dynamical behavior as has hitherto been discussed.

\subsubsection{Embedding dimension}

The computation of the embedding dimensions, i.e. the number of dimensions in which the attractor of the assumed dynamical system lies, is vitally important for the phase space reconstruction. Thereby, the attractor of the underlying dynamical system is assumed to fold and unfold smoothly when the embedding changes from an integer value to the next one, without sudden irregularities in its structure. A popular method for estimating a proper embedding dimension for the dynamical system's attractor, denoted by $\mu$, is the false nearest neighbor [Kennel et al., 1992] method. With this method, the unfolding smoothness of the attractor is examined in spaces with increasing dimension

\footnotetext{
${ }^{1}$ Several other considerations of polyphonic events were considered, like from lowest to highest tone, selecting only the highest tone, and random selection of tone order in polyphonic events, and the results were similar, and therefore omitted in the present study.
} 
( $\mu$ values). Thus, for each reconstructed point in the examined embedding dimensions, $\mu$, it is investigated whether its nearest neighbor remains below a distance threshold when the embedding dimension is increased to $\mu+1$. If this does not hold, then these points are called false neighbors. The embedding dimension that is chosen as appropriate, is the one that incorporates a small percentage of false neighbors when the dimension is increased. In the context of the presented work, the satisfactory percentage of false nearest neighbors was set to zero. Therefore, the proper embedding dimension was chosen to be the smallest one that incorporates no false nearest neighbors.

\subsection{Largest Lyapunov exponent}

The sensitivity of a dynamical system to initial conditions is quantified by the Lyapunov exponents, which roughly discuss the expansion or contraction rate of trajectories with initial conditions over a small $\mu$-dimensional sphere. While the system's iterations progress, the sphere evolves into an ellipsoid the principal directions of which expand, contract or remain unchanged, as measured by positive, negative or zero Lyapunov exponents. In directions which incorporate negative Lyapunov exponents, the attractor's projection is a fixed point, while zero Lyapunov exponents denote directions of limit circles. Positive Lyapunov exponents signify the existence of chaos. The set of Lyapunov exponents to all directions is called the Lyapunov spectrum and it is denoted by $\left(\lambda_{1}, \lambda_{2}, \ldots, \lambda_{\mu}\right)$, but random initial condition vectors are expected to converge to the most unstable manifold (with probability 1 [Rosenstein et al., 1993]). Therefore, the computation of the largest Lyapunov exponent (LLE), $\lambda_{1}$, is sufficient to indicate the existence of chaos in the examined system.

Although there are numerical methods for computing the entire Lyapunov spectrum [Wolf et al., 1985] from time series, the acquisition of the largest exponent is needed to define the attractor's chaotic potentiality. A well-known methodology for evaluating the LLE of the reconstructed attractor from a time series, is due to Rosenstein [Rosenstein et al., 1993] and is the numerical method utilized in the paper at hand. A prerequisite for Rosenstein's method is to reconstruct the attractor's phase space based on the time series observation, thus obtaining the time delay $(\tau)$ and embedding dimension $(\mu)$ as described in Sec. 2.1. After the reconstruction, the nearest neighbor $\left(\mathbf{y}_{\hat{\imath}}\right)$ of each point $\left(\mathbf{y}_{i}\right)$ in the trajectory is located and their distance in this initial iteration is computed as

$$
d_{i}(0)=\min _{\mathbf{y}_{\hat{\imath}}}\left\|\mathbf{y}_{i}-\mathbf{y}_{\hat{\imath}}\right\|_{2} .
$$

The LLE is then estimated as the mean rate of nearest neighbor separation throughout their successive iterations. By definition, therefore, it holds that

$$
d_{i}(t)=d_{i}(0) \mathrm{e}^{\lambda_{1}(t)},
$$

where $\lambda_{1}(t)$ is the LLE estimation at time step $t$. Taking the logarithm of both sides in the equation, we have

$$
\ln \left(d_{i}(t)\right)=\ln \left(d_{i}(0)\right)+\lambda_{1}(t) .
$$

The average LLE is finally approximated by the gradient of the linear regression among all neighboring pairs

$$
b(t)=\left\langle\ln \left(d_{i}(t)\right)\right\rangle_{i},
$$

where $\langle\cdot\rangle_{i}$ denotes the mean value over all possible $i$ indexes.

\subsection{Fractal dimension}

Fractal dimension (FD) is a concept that describes the complexity of geometric objects as a ratio of change in detail to the change in scale [Mandelbrot, 1982]. Unlike the topological dimension, FD may have noninteger values and a popular method for its computation is the well-known box counting method [Alevizos \& Vrahatis, 2010]. Several methods have been proposed for the FD computation of time series, among which are the correlation dimension method [Grassberger \& Procaccia, 1983], the methods of Kantz [1988], Higuchi [1988], Petrosian [1995] and Sevcik [1998]. Many works have compared the above methodologies either by their effectiveness on experimental data (for example, for seizure detection in EEG), and/or on functions with theoretically computable FD values (like the Weierstrass cosine functions [Tricot, 1994]) [Esteller et al., 2001; Goh et al., 2005; Raghavendra \& Dutt, 2010; Ahmadi \& Amirfattahi, 2010; Polychronaki et al., 2010]. The results yielded by these works provide uncertain and contradicting results about the suitability of each method for different tasks, reflecting the fact that the computation of the FD of time series is case dependent.

The method used for the results presented in this paper is the method of Sevcik [1998], since 
it is among most easily implementable and faster methods. This method is based on the computation of

$$
D_{H}=\lim _{\varepsilon \rightarrow 0} \frac{-\log (N(\varepsilon))}{\log (\varepsilon)},
$$

where $N(\varepsilon)$ is the number of $n$-dimensional open balls with radius $\varepsilon$ needed to completely cover the set under examination. If a length $L$ is assumed for the set that comprises the curve under examination, then this curve can be covered by at least $N(\varepsilon)=\lceil L /(2 \varepsilon)\rceil$ balls of radius $\varepsilon$. Sevcik proposed the consideration of the time series as a twodimensional object, laying on the plane defined by time ( $x$-axis) and the time series' values ( $y$-axis). Therefore, the balls that are utilized for the examination of the curve coverage, are considered as two-dimensional balls. With these facts under consideration, the computation of $D_{H}$ may be written as [Raghavendra \& Dutt, 2010]

$$
D_{H}=1-\lim _{\varepsilon \rightarrow 0} \frac{\log (L)-\log 2}{\log (\varepsilon)} .
$$

The method proposed by Sevcik utilizes the latter expression of $D_{H}$ to derive the counterpart computation for time series. Thereby, if a single dimensional time series is considered, a normalization of both the time series values $\left(y_{i}\right)$ and indexes $\left(x_{i}\right)$ is considered, with $y_{i}^{*}=\left(y_{i}-y_{\min }\right) /\left(y_{\max }-y_{\min }\right)$ and $x_{i}^{*}=\left(x_{i}-x_{\min }\right) /\left(x_{\max }-x_{\min }\right)$ respectively, where $y_{\min }, y_{\max }, x_{\min }$ and $x_{\max }$ are the minimum and maximum values and indexes respectively. By considering a time series with $N$ observations, an $N \times N$ grid is constructed on the normalized $x y$ time series plane, and the fractal dimension is approximated as [Raghavendra \& Dutt, 2010]

$$
D_{s}=1+\frac{\log (L)-\log (2)}{\log (2(N-1))}
$$

where $L$ is the length of the normalized time series.

\section{Automatic Composition of Tonal Sequences with Specified Characteristics}

Automatic symbolic music composition encompasses a great variety of methodologies that produce novel music content in the form of tones, onsets, note durations and timbre among others. The specific subdomain that the paper at hand discusses could be characterized as "supervised" algorithmic composition, in a sense that the compositional algorithm is "forged" to compose music which complies with certain musical characteristics. Under this perspective, the composition process is integrated with a supervised training process, in which the parameters of the automatic music generation algorithm are properly adjusted, to allow the composition of music that is circumscribed within a musical area of predefined characteristics. In the context of the presented work, the algorithm that generates notes comprises an iterative map among the ones demonstrated in Table 1 and a typical iteration-totones interpretation methodology that is described in Sec. 3.3.

The tunable parameters of the automatic composition algorithm are the parameters of the maps, within the ranges demonstrated in the third column of Table 1. It has to be noticed that the initial conditions of a map is also considered as a parameter in the present work. Parameter values within the selected ranges, allow the respective maps to expose their dynamic potentialities, from fixed points and periodic orbits to chaos. These parameters are properly adjusted so that the generated tones comply with some musical criteria that are defined by a set of music features. The adjustment process is realized with the Differential Evolution (DE) algorithm [Storn \& Price, 1997; Price et al., 2005], which evolves populations of parameter values towards values that are better fitted to the problem at hand, i.e. they allow the respective iterative systems to compose music that complies with the target music features. The target music features are extracted from each piece in the set of available genuine pieces. Under the guidance provided by a target genuine piece in the form of music features, each map generates a new composition which is expected to have similar features to the ones provided by the target piece. The rhythmic and orchestration part of music are not considered and therefore, the notes produced by the dynamical systems are "attached" to the respective rhythmic values of the target pieces.

\subsection{Evolving dynamical systems to composers}

The appropriateness of a map's parameters, considering the established evolutionary nomenclature, is called "fitness" and it is a value that describes the "distance" between the features of the target and the artificial composition. A proper distance 
Table 1. The iterative maps used as tone generators, their parameters and the parameters' considered ranges.

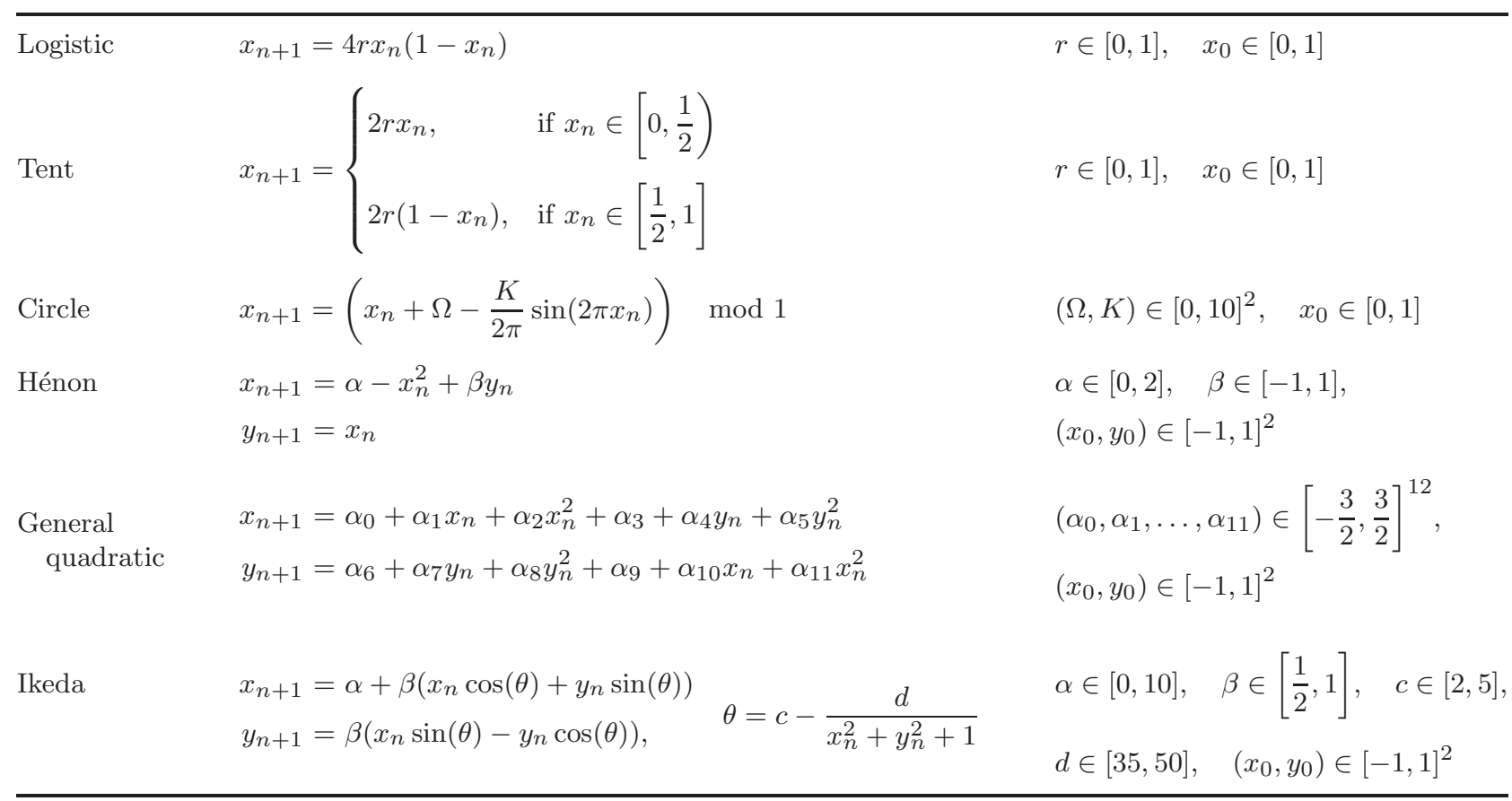

measure should consider all features equally important, to drive the evolution of new dynamical system parameters towards ones that compose better music, according to every feature impartially. Since the range of the features is not known a priori, or it could be any value in $(-\infty, \infty)$, a straightforward normalization of the feature values in $[-1,1]$ is not possible. To this end, the mean relative distance, denoted $d_{\mathrm{MRD}}$ measure could provide a good impartial approximation of the feature differences, since it offers an estimation about the percentage of the difference between the features of the genuine and the artificial pieces. Considering two vectors of features, $\mathbf{f}_{\mathrm{g}}$ and $\mathbf{f}_{\mathrm{a}}$, their mean relative distance is computed as [Kaliakatsos-Papakostas et al., 2013]

$$
d_{\mathrm{MRD}}=\frac{1}{k} \sum_{i=1}^{k} \frac{\left|\mathbf{f}_{\mathrm{g}}(i)-\mathbf{f}_{\mathrm{a}}(i)\right|}{\max \left(\left\{\mathbf{f}_{\mathrm{g}}(i), \mathbf{f}_{\mathrm{a}}(i)\right\}\right)},
$$

where $k$ is the length of the feature vectors and denotes the number of features, the index $i$ refers to the $i$ th vector element, or the $i$ th feature and $\max (a, b)$ returns the maximum among the numbers $a$ and $b$. A detailed description of the music features that comprise the music vector is provided in Sec. 3.2.

Each iterative map listed in Table 1 is employed as a music composer that generates tonal sequences with a methodology described in Sec. 3.3. The characteristics of the music it composes rely on its parameters' values. Therefore, a method that extensively searches for proper parameter values is required, in order to explore the map's compositional potentialities comprehensively. As mentioned earlier, this method is the DE algorithm. With this method, a population of parameter values (called individuals) is randomly initialized, and the system is allowed to compose music with each one of them. Afterwards, these parameters are altered with the application of some evolutionary operators, which create new individuals (i.e. sets of parameter values). If the new individual produces "better music" than its ancestor, then it is selected to belong to the new generation of individuals, else its ancestor passes on to the new generation. This procedure continues iteratively, creating populations of parameter values with better fitness, i.e. they produce better music. For the presented results, a population size of 50 individuals was utilized and evolved for 50 generations. The range of the parameter values throughout all iterations, were forced to remain within the limits demarcated in the third column of Table 1 respectively for each map.

\subsection{Tonal features in short music segments}

When considering automatic music composition towards the direction provided by some music 
Table 2. The considered tonal features.

\begin{tabular}{lll}
\hline \multicolumn{1}{c}{ Name } & Acronym & \multicolumn{1}{c}{ Description } \\
\hline Tonal range & range & Difference between maximum and minimum pitch values \\
Tonal gradient & grad & Gradient of the interpolating line through pitch values \\
Tonal jumps mean & pdM & Mean value of consecutive pitch value differences \\
Tonal jumps standard deviation & pdS & Standard deviation of pitch value differences \\
Ascending profile & asc & Ratio of ascending intervals over the total number of intervals \\
Descending profile & desc & Ratio of descending intervals over the total number of intervals \\
Constant profile & const & Ratio of constant intervals over the total number of intervals \\
\hline
\end{tabular}

features, these features should encompass "fundamental" music attributes, which describe as accurately as possible the desired music output. Furthermore, features that describe musical characteristics accurately should yield clearer comparison results, allowing safer determination about which map exhibits better compositional capabilities. The features that have been selected for the reported results are shown in Table 2. Since the purview of this paper is the tonal musical domain (not rhythm or orchestration), the features are focused on tonal aspects, which concern statistics about the transition of notes. These features do not incorporate statistics about the tonal constitution of pieces, i.e. statistics that reflect the key of composition, since such features would not contribute to the assessment of the automatic composition system's adaptability.

To obtain an insight about the musical descriptiveness of the seven aforementioned music features, a two-sided Wilcoxon [Wilcoxon, 1945] rank sum test is applied on the features extracted by short segmentations of each piece for each composer. Through this test, the statistical significance of the difference in each feature's distribution for each composer is examined. Specifically, for each pair of composers we employ the rank sum test to each respective feature pair, to obtain the probability that these two features belong to continuous distributions with equal medians, a fact that would not make them descriptive. Formally, the null

Table 3. Statistical significance in the difference of each feature's distributions among the pieces of each composer.

\begin{tabular}{lcccc}
\hline & Bach & Mozart & Beethoven & Jazz \\
\hline Bach & - & 1101101 & 1111111 & 1111111 \\
Mozart & - & - & 1101111 & 1111111 \\
Beethoven & - & - & - & 1111111 \\
\hline
\end{tabular}

hypothesis for each feature pair is that they are independent samples from identical continuous distributions with equal medians. If the null hypothesis is rejected at the $5 \%$ significance level for a pair of composers and a pair of features, then these features are indicated to encompass significant different statistical information about each composer.

The results of this test are demonstrated in Table 3. Each cell in this table concerns the results about each composer pair. The sequence of binary digits within each cell denotes the rejection of the null hypothesis with 1 and contrarily with 0 , for the respective feature. For example, for the BachMozart pair, the null hypothesis is rejected to all but the third and sixth features. Therefore, each quadruple of 1 and 0 digits denotes the significance or not respectively, of distribution differences between composer pairs for the respective feature. For example, the sequence 1101111 for the MozartBeethoven pair denotes that the distributions of all features except the third are significantly different. Since Table 3 exhibits a statistical significance in the distributions of most pairs of respective features for each composer, it is clearly indicated that these features encompass viable musical information. Therefore, within the extent of the presented statistical analysis, the results that are reported in Sec. 4 could be trusted as indicative of the compositional capabilities of each dynamical system.

\subsection{Automatic tonal composition methodology}

Since the main subject of examination is the tonal compositional potentialities of iterative dynamical systems, the automatic composition process is directed solely towards tonal composition. The rhythmic elements are considered to be borrowed by the target piece, thus creating a composition with identical rhythm. The specific scope of the 
presented tonal composition methodology, assumes the existence of a target genuine music piece which provides the tonal compositional guidelines for the generative system. It is important that these guidelines encompass the necessary amount of information, which may be assumed by general admissions. For instance, although it is a tempting conjecture, one may hardly presume that a dynamical system would form musical structures that resemble human compositions in every hierarchical level, e.g. key structure, chord progressions or note motifs. The fact that musical knowledge is not inherent to dynamical systems has been addressed by several means, like the imposition of key constraints [Coca et al., 2010; Kaliakatsos-Papakostas et al., 2012b] (i.e. eligible notes on certain key) or tonal restrictions imposed by a target piece itself [Dabby, 1996].

A tone generation approach similar to the one presented here has been utilized for the generation of tonal sequences, in the context of an automatic intelligent music improviser [KaliakatsosPapakostas et al., 2012a]. The compositional methodology used in the current work is adjusted to receive tonal guidelines from the target piece, while the above cited system receives guidelines from a human improviser. The target piece provides guidelines that encompass music knowledge at an elementary level, within short segments throughout its duration. These guidelines incorporate tonal range, chord information and pitch class profile (PCP) complexity expressed by the Shannon information entropy [Shannon, 2001] (SIE). These information features characterize a list of allowed notes, which are eligible to be "played" by the dynamical system through a straightforward mapping procedure. An overview of the system, as described in this paragraph, is illustrated in Fig. 1, while a detailed analysis is provided in the next two paragraphs.

The target piece provides some intrinsic musical guidelines to the dynamical system, which characterize the target piece's music content in short time segments, through the formulation of a note list for each segment. The note list comprises notes within the range of each respective segment, which also belong to certain pitch class values. Initially, the target genuine piece is segmented in short intervals, according to its tempo, and the chord of each segment is recognized with a typical template-based technique [Oudre et al., 2010], considering several chord templates with up to five voices. Since the available pieces are in MIDI format, a segmentation
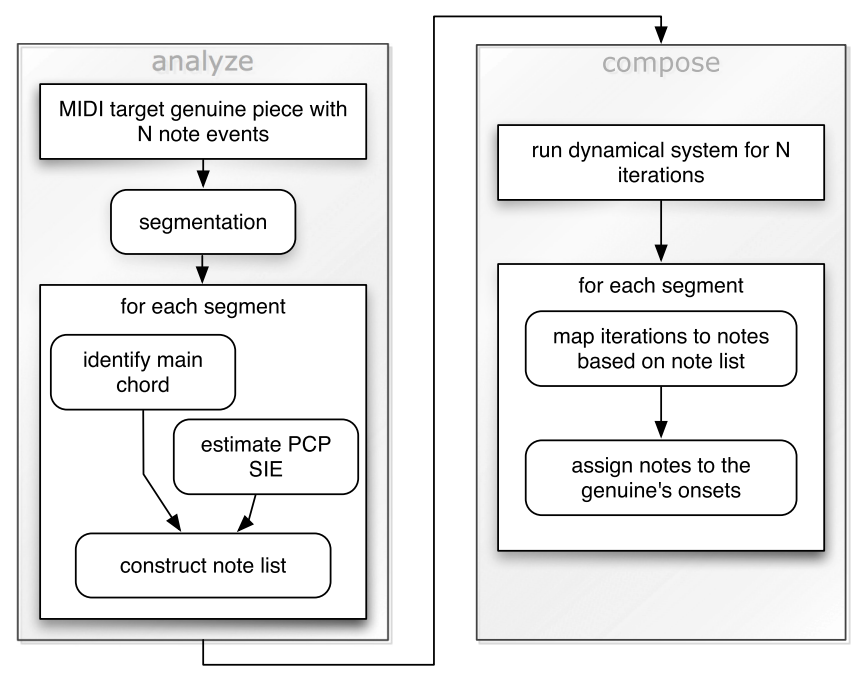

Fig. 1. Flow diagram of the automatic composition process.

in certain measure subdivisions is possible. To this end, a segment length of two beats was considered the most appropriate compromise between selecting a too small, or a too large segmentation length. The main concern that the discussed methodology faces is the detection of chords within each segment. Therefore, too small a segment may not provide a sufficient amount of notes for successful chord recognition. Contrarily, larger segments would most likely incorporate more than one chord, leading, in the best case, to the disregard of all except one chord in the segment.

At first, the note list of each segment includes the notes that pertain in the recognized chord, but additional notes may be required to capture the tonal constitution of a segment. No matter what segmentation length would be chosen, it would still be possible to find a segment which incorporates more than one chord, or a segment with no chord (e.g. chromatic phrase). To this end, the supplementary information provided by the SIE of the PCP is also considered. In each segment, a value is obtained from the SIE of the PCP, which indicates the information complexity of tones in this segment. If this complexity is "reachable" by utilizing only notes of the recognized chord, then the notes that comprise the chord are sufficient and no additional notes are needed to form the final note list of the segment. On the contrary, if the SIE threshold of the recognized chord is exceeded, the most prominent notes of the PCP are added incrementally to the final note list, except from the ones that are already added from the recognized chord, until the 
SIE threshold is covered. The final list of available notes comprises notes within the range dictated by the respective segment and belong to the recognized chord, with possible additional notes imposed by the SIE complexity threshold. After the note list has been constructed, the iterations of the dynamical system are normalized within $[0,1]$ and are then linearly mapped to the indexes of each segment's note list. The tonal sequences that are produced, are adhered to the rhythmic note events of the genuine composition.

\section{Results}

The results focus on three inquiries. At first, the dynamical properties of the genuine compositions are assessed, providing information about the attractor characteristics of each composer's tonal sequences. Secondly, results are reported on the adaptivity of each dynamical system to the tonal characteristics that are imposed by each composer. This analysis incorporates a thorough examination of the musical characteristics that each system is able to reproduce. Finally, a comparative analysis is performed on the dynamical characteristics of the genuine and the artificial compositions.

\subsection{Time series analysis on the genuine target compositions}

This section presents the findings yielded by the application of the methods presented in Sec. 2 . Specifically, the note sequence of each genuine piece is considered to form a time series, by which the information of the attractor reconstruction are assessed, namely the time delay $(\tau)$ and embedding dimension $(\mu)$, together with the Largest Lyapunov Exponent (LLE) and the Fractal Dimension (FD). This analysis is supplementary to the main perspective of the paper, which is the examination of the compositional capabilities of several dynamical systems. Therefore, this analysis firstly aims to provide some descriptive statistical information about the attractor characteristics for different composers through the $\tau, \mu$, LLE and FD values assessed by every piece. Secondly, the LLE and FD values offer the opportunity to perform a comparative analysis, presented in Sec. 4.3, between the respective values extracted from the pieces that were artificially produced by the dynamical systems.

The attractor characteristics are shown in the box plots in Fig. 2. Although the values presented therein constitute numerical approximations, it is clearly observed that there are differences of these characteristics between pairs of composers. To examine which time series characteristics are significantly different between which pairs of composers, we perform a two-sided Wilcoxon [Wilcoxon, 1945] rank sum test, in a similar fashion as with the music features in Sec. 3.2. For the currently examined case, the null hypothesis is that the observations deriving from a time series' values from a composer pair, are samples from identical continuous distributions with equal medians. The results for these tests are demonstrated in Table 4, while it is reminded that the examined pieces are all piano compositions or piano transcriptions (in the case of the jazz standards). Therein, each four-tuple of digits denotes whether the null hypothesis is rejected for the distributions of the respective time series values, for the respective composer of each row and column. Specifically, the digit 1 shows the rejection of the null hypothesis on the significance level of $5 \%$ (thus the results are statistically significant), while the digit 0 the opposite. In specific, each quadruple of 1 and 0 digits denotes the rejection or not respectively, of the null hypothesis for the distribution of $\tau, \mu$, LLE and FD respectively between composer pairs. For example, the sequence 1101 for the BachMozart pair denotes that the differences in $\tau, \mu$ and FD distributions are statistically significant, while the LLE are not. The display order of each digit corresponds to the distributions of $\tau, \mu$, LLE and FD values. It should be noted that for every pair of composers, there is at least one pair of distributions that is significantly different, while the opposite holds for the LLE distributions.

\subsection{Adaptivity of each dynamical system}

The inquiries discussed in this paragraph incorporate the adaptivity of each dynamical system to the specified composition tasks. The adaptivity is measured by the fitness value of the best individual yielded by the evolutionary process as described in Sec. 3.1. Table 5 presents the mean value and the standard deviation of the fitness values provided by the best individual in each composition task. It is reminded that each individual represents a set of parameter values (including initial iteration) for each map, as displayed in the third column of Table 1, and that a composition task is the composition of novel tonal content that encompasses the 




(a)



(c)

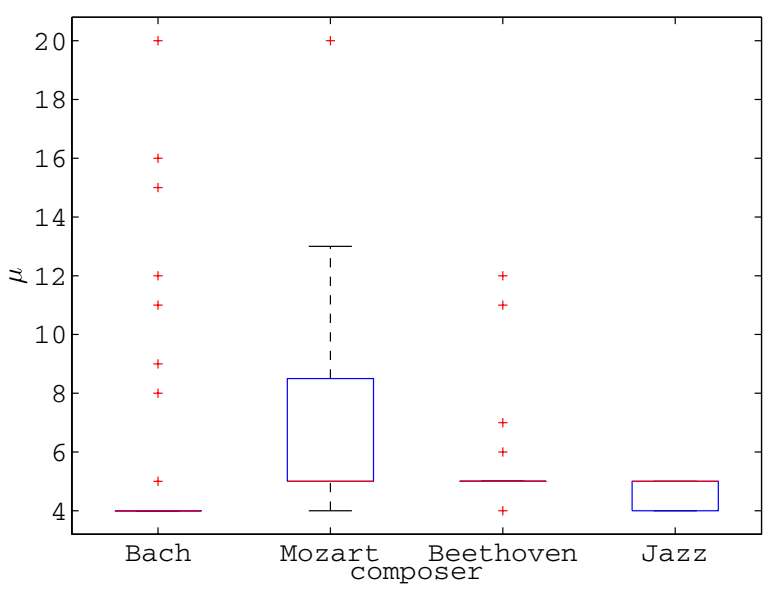

(b)

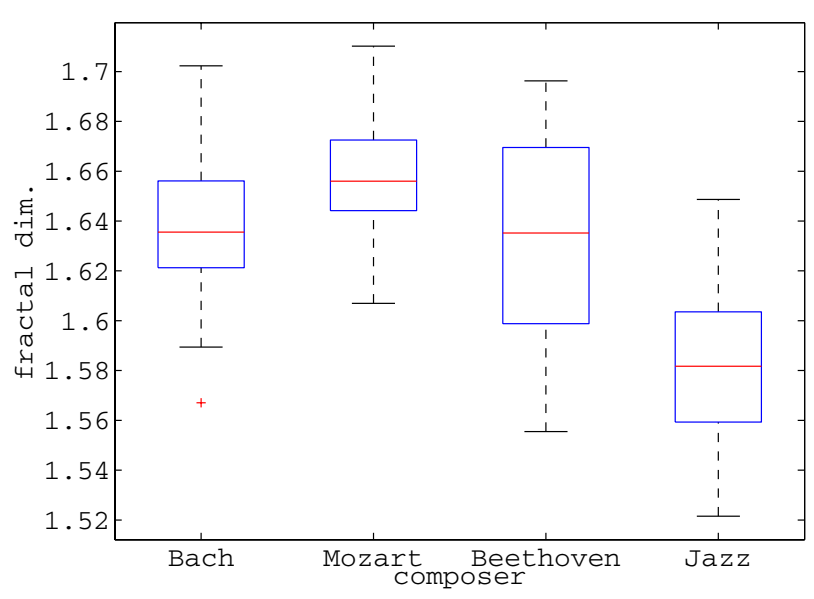

(d)

Fig. 2. Estimated (a) $\tau$, (b) $\mu$, (c) LLE and (d) FD values for the tonal time series of each piece in the dataset.

tonal characteristic of a target piece. From Table 5 one may first notice that the worst performance for all composers is provided by the tent map, while the general quadratic map has produced individuals with the best mean fitness, for the compositions of all composers except from Beethoven. The best mean performance for Beethoven was achieved by the circle map. Nevertheless, the mean performance of the general quadratic, the circle and the Hénon

Table 4. Statistical significance of differences between $\tau, \mu$, LLE and FD distributions among the pieces of each composer.

\begin{tabular}{lcccc}
\hline & Bach & Mozart & Beethoven & Jazz \\
\hline Bach & - & 1101 & 1100 & 0101 \\
Mozart & - & - & 0001 & 1101 \\
Beethoven & - & - & - & 1101 \\
\hline
\end{tabular}

maps among all composers are comparable. A more thorough quantification of the significance of this similarity is performed below.

Figure 3 depicts the distribution of the training errors among the examined music features with error bars, for the best and worst performing dynamical systems. This figure signifies that the main differences of performance in these systems lie on the first and the last three features, namely the range and percentages of ascending, descending and constant intervals. An additional fact that should be noticed is the arithmetic value of the mean fitness errors. For almost any measurement this value lies between 0.2 and 0.3 , while even the best mean value is above 0.2 . By considering the fitness measurement method, the mean relative distance (MRD) as defined in Sec. 3.1, one may assume that the best artificial compositions are expected to be $20 \%$ different than the original 
Table 5. Mean and standard deviation (in parentheses) of the best individual's fitness values for all composers and maps. The best mean fitness value for each composer is demonstrated in boldface typesetting.

\begin{tabular}{lcccccc}
\hline & Logistic & Tent & Circle & Hénon & Gen. Quad. & Ikeda \\
\hline Bach & $0.237(0.017)$ & $0.268(0.017)$ & $0.213(0.023)$ & $0.217(0.017)$ & $\mathbf{0 . 2 1 2}(0.021)$ & $0.229(0.020)$ \\
Mozart & $0.279(0.021)$ & $0.306(0.015)$ & $0.257(0.022)$ & $0.260(0.024)$ & $\mathbf{0 . 2 5 4}(0.024)$ & $0.274(0.023)$ \\
Beethoven & $0.261(0.021)$ & $0.277_{(0.020)}$ & $\mathbf{0 . 2 5 5 ( 0 . 0 1 6 )}$ & $0.256(0.018)$ & $0.257(0.022)$ & $0.268(0.018)$ \\
Jazz & $0.276(0.015)$ & $0.295(0.015)$ & $0.264(0.020)$ & $0.263(0.016)$ & $\mathbf{0 . 2 5 8 ( 0 . 0 1 6 )}$ & $0.271(0.013)$ \\
\hline
\end{tabular}

ones, if such a lax quantitative conclusion may be reached. When consulting the findings in Fig. 3, however, it is indicated that there is a malapportionment of the errors among different features. Specifically, the grad feature, which is the gradient of the line that interpolates the notes in a segment, presents errors around $100 \%$.

This big difference in the gradients may be explained by consulting Fig. 4, which illustrates the mean and standard deviation of the artificial pieces' tonal features. The values of the gradients are exhibited to be small in magnitude, a fact that also holds for the respective feature in the genuine compositions. Therefore, an endogenous imbalance of the MRD is exposed when considering small magnitudes. For example, if the target gradient in a segment is 0.001 and the dynamical system generates music that presents a gradient of 0.02 in the respective segment, then the MRD value is 0.95 , denoting $95 \%$ error. This measurement is in contradiction with the intuitive approach that both gradients are small in magnitude. The errors in the rest of the features remain in levels compared with the overall fitness (around 0.2 or 20\%), with an exception in the range feature where better performance was reached for both the best and the worst performing maps, although their difference is noticeable. Better performance for this feature is expected, since the range is considered in the construction of the note list, as discussed in Sec. 3.3. Therefore, accurate measurements for this feature could be assessed if the iterations of the dynamical systems approached their entire range in every segment.

Figure 4 also demonstrates that the worst performing map generates tones which efface the characteristic tonal marks of each composer, as imprinted in the collected features. Contrarily, the best performing map preserves, at some extent, the uniqueness of features for each composer. Therefore, the implications of fitness difference are also indicated to entail a "homogenization" in the qualitative and discriminative characteristics of the generated music. This raises a question of which map captures each music characteristic, expressed by the examined features, more successfully. This inquiry is scrutinized in Table 6 with a comparative analysis between all pairs of maps, for each feature and for every composer. Therein, the dynamical system of each row is compared to the system of each column according to their performance in each feature, for each composer. If the map of a row performs significantly better than the map of a column for a specific feature and composer, then a "+" sign appears in the respective row, column, composer

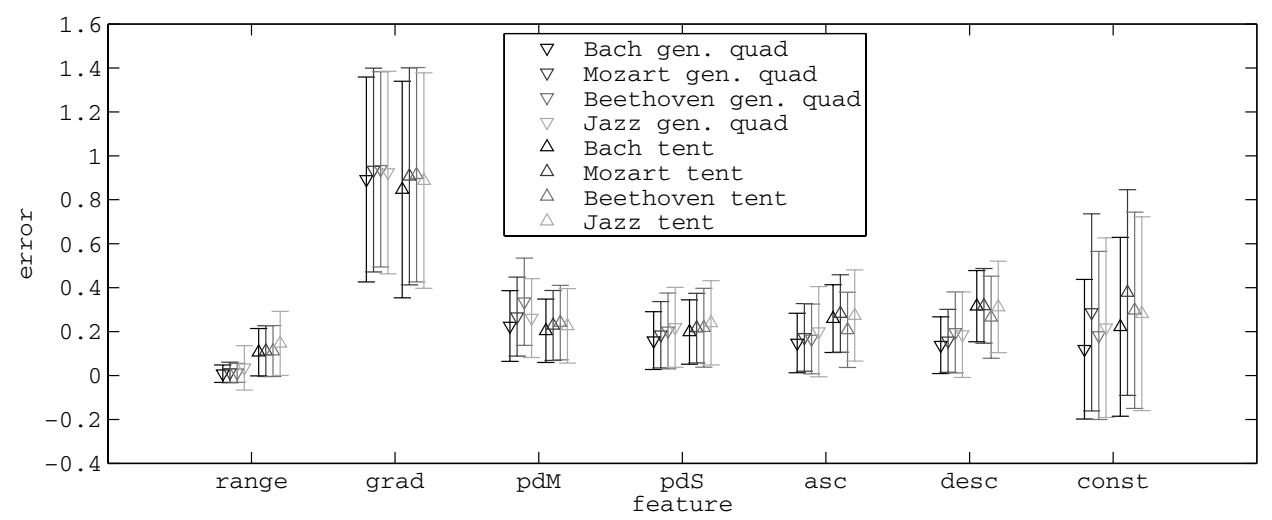

Fig. 3. The errors of each feature for the best (general quadratic) and the worst (tent) overall fitted maps. 


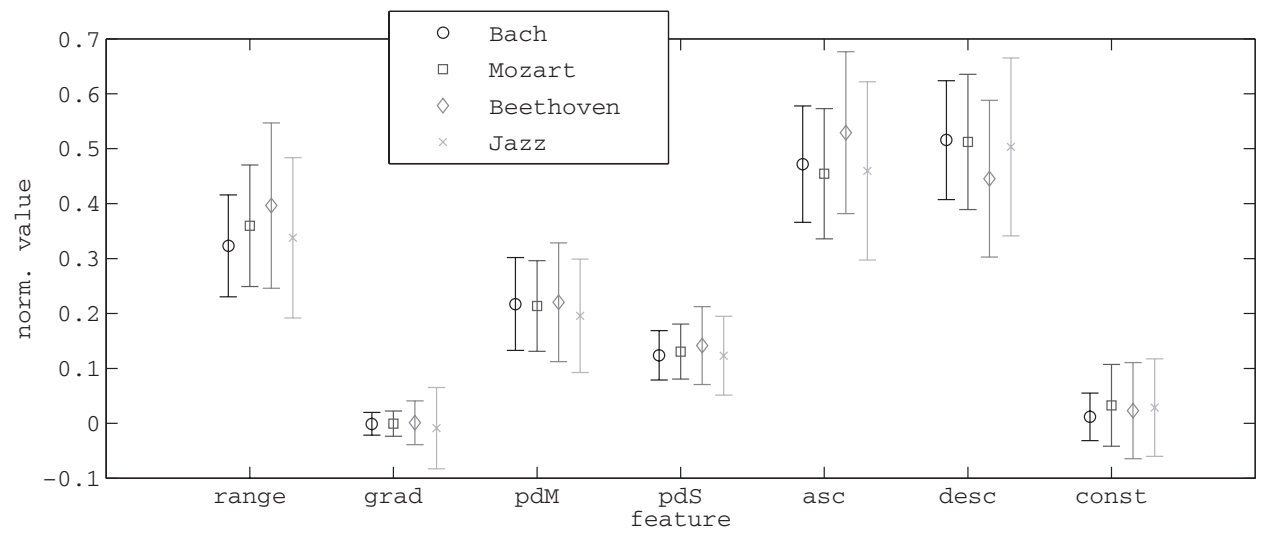

(a)



(b)



(c)

Fig. 4. Error bars of normalized feature distributions for each composer, (a) for genuine composition, (b) for artificial ones produced by the general quadratic map and (c) by the tent map.

and feature order. The results for each composer appear in each line in the respective cell (with the order being (Bach, Mozart, Beethoven and Jazz)), while the features are presented from left to right in the order appeared in Table 2. If the row map is outperformed significantly, this is denoted with a "-" sign, while a "=" sign denotes that there is no significant difference.

The significance in error differences is measured with the two-sided Wilcoxon rank sum test, as previously, with the null hypothesis being that the errors produced by a map for every composer and 
Table 6. Statistical significance of the compositional superiority of each map according to each feature and composer. Each cell in the table incorporates the comparison of the maps in the respective row and column. A significant superiority of the row map per feature and composer is denoted by the "+" sign, the opposite with the "-" sign, while no significant difference with the "=" symbol. Each row of symbols in a cell represents the pieces of each composer.

\begin{tabular}{|c|c|c|c|c|c|c|}
\hline & Logistic & Tent & Circle & Hénon & Gen. Quad. & Ikeda \\
\hline Logistic & - & $\begin{array}{l}+-=++++ \\
+--++++ \\
+=-++++ \\
+--=++-\end{array}$ & $\begin{array}{l}-=-+--= \\
-=-+--= \\
-=-+=-= \\
---=---\end{array}$ & $\begin{array}{c}-=+=--- \\
-=+=--= \\
-===--= \\
--+----\end{array}$ & $\begin{array}{l}-=+---- \\
-=+---= \\
-++=--= \\
-=-----\end{array}$ & $\begin{array}{l}-=++--= \\
-=++--= \\
-++=--= \\
--+----\end{array}$ \\
\hline Tent & - & - & $\begin{array}{l}-=----- \\
-=+---- \\
-=++--- \\
-=+=---\end{array}$ & $\begin{array}{l}-++---- \\
-++---- \\
-=+=--- \\
-++----\end{array}$ & $\begin{array}{l}-++---- \\
-++---- \\
-++---- \\
-++----\end{array}$ & $\begin{array}{l}-++---- \\
-++---- \\
-=+---- \\
-++----\end{array}$ \\
\hline Circle & - & - & - & $\begin{array}{l}-++-==- \\
-=+-++- \\
-=+-==- \\
-++-==-\end{array}$ & $\begin{array}{l}-++-==- \\
-=+---- \\
-=+-==- \\
-+=----\end{array}$ & $\begin{array}{l}+++=== \\
+=+=++= \\
+=+---= \\
-=+----\end{array}$ \\
\hline Hénon & - & - & - & - & $\begin{array}{l}+=-=== \\
+=----= \\
+=+==-= \\
+=-=--=\end{array}$ & $\begin{array}{l}+=++==+ \\
+=++--= \\
+=+=--+ \\
+=+=-=+\end{array}$ \\
\hline Gen. Quad. & - & - & - & - & - & $\begin{array}{l}+=++==+ \\
+=++++= \\
+=+===+ \\
+=+====\end{array}$ \\
\hline
\end{tabular}

each feature belong to identical continuous distributions with equal medians. The rejection of the null hypothesis in the $5 \%$ significance level for each respective pair of error distributions is denoted with a "+" or "-" and the contrary with the "=" symbol. The findings in Table 2 demonstrate that there is an overall feature superiority for the better performing maps, with some exceptions mainly encountered for the second and third features (the tonal range and mean value of consecutive pitch differences). This fact probably signifies that the dynamical systems with the "weakest" compositional skills were the ones that could not produce descending, ascending and constant patterns that resembled the genuine compositions. Therefore, the evolutionary process endued these maps with the comparative advantage to compose music with more accurate overall tonal gradient and between-pitch distances.

\subsection{Dynamical properties of the genuine and the artificial compositions}

As mentioned in Sec. 2, a comparison of the dynamical properties of the artificial compositions is also examined. The dynamical characteristics incorporate the LLE and the FD. The phase space reconstruction is not necessary since its properties are already known through the analytic form of the dynamical systems. Furthermore, since the dynamical systems are known, different approaches could be followed for the computation of the LLE and FD (probably including analytic computation where permitted). Nonetheless, since a comparative analysis is pursued, the same numerical approaches were considered as for the genuine compositions. Therefore, the tonal parts of the artificial compositions were considered themselves as time series, with their attractor characteristics (time delay and embedding dimension) being provided by the respective dynamical system that produced them. Through these time series, the LLE and FD were extracted and the results, together with the fitness values, are illustrated with box plots in Fig. 5. These results are grouped in two manners, according to composer and map.

The finding in Figs. 5(c) and 5(e) demonstrate the distributions of the LLE and FD for all the artificial compositions of all maps. In comparison to the respective distributions in Figs. 2(c) and 2(d), the 
Chaos and Music: From Time Series Analysis to Evolutionary Composition

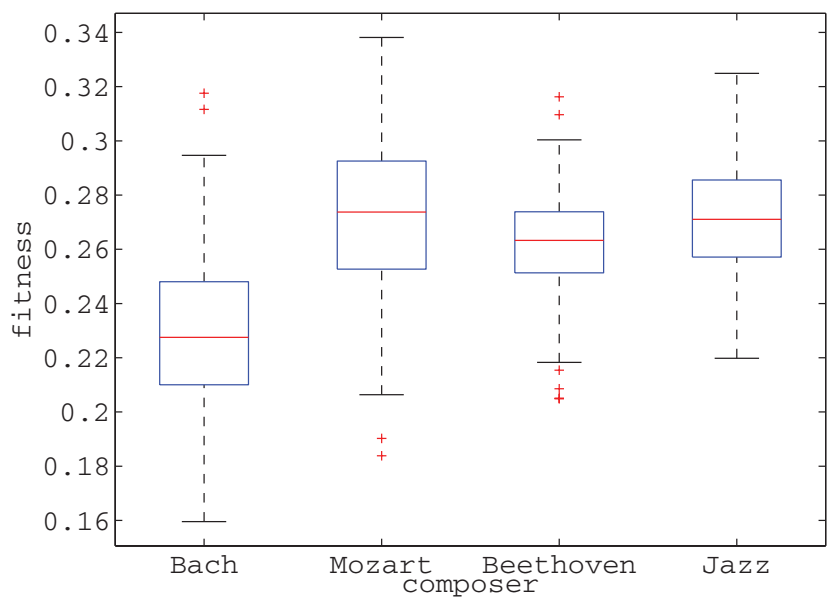

(a)



(c)



(e)



(b)



(d)



(f)

Fig. 5. Fitness, LLE and FD per composer and per map for the artificial compositions. (a) Fitness per composer, (b) fitness per map, (c) Lyapunov per composer, (d) Lyapunov per map, (e) FD per composer and (f) FD per map. 
Table 7. Mean and standard deviation (in parentheses) of the best individual's largest Lyapunov exponent values for all composers and maps. The values that correspond to best mean fitness value in Table 5 are demonstrated in boldface typesetting.

\begin{tabular}{lccccccc}
\hline & Genuine & Logistic & Tent & Circle & Hénon & Gen. Quad. & Ikeda \\
\hline Bach & $0.043(0.105)$ & $0.373(0.211)$ & $0.627(0.016)$ & $0.374(0.457)$ & $0.076(0.096)$ & $\mathbf{0 . 0 7 0}(0.107)$ & $0.287(0.169)$ \\
Mozart & $0.021(0.031)$ & $0.305(0.189)$ & $0.629(0.021)$ & $0.370(0.496)$ & $0.082(0.105)$ & $\mathbf{0 . 0 2 9}(0.060)$ & $0.327(0.197)$ \\
Beethoven & $0.053(0.163)$ & $0.345(0.214)$ & $0.626(0.015)$ & $\mathbf{0 . 2 5 0 ( 0 . 2 9 6 )}$ & $0.089(0.147)$ & $0.074(0.109)$ & $0.269(0.231)$ \\
Jazz & $0.146(0.302)$ & $0.311_{(0.191)}$ & $0.624(0.019)$ & $0.533(0.537)$ & $0.057(0.091)$ & $\mathbf{0 . 0 2 2}(0.031)$ & $0.364(0.202)$ \\
\hline
\end{tabular}

Table 8. Mean and standard deviation (in parentheses) of the best individual's fractal dimension values for all composers and maps. The values that correspond to best mean fitness value in Table 5 are demonstrated in boldface typesetting.

\begin{tabular}{lccccccc}
\hline & Genuine & Logistic & Tent & Circle & Hénon & Gen. Quad. & Ikeda \\
\hline Bach & $1.637(0.027)$ & $1.721(0.013)$ & $1.685(0.004)$ & $1.701(0.021)$ & $1.730(0.017)$ & $\mathbf{1 . 7 1 4}(0.022)$ & $1.732(0.008)$ \\
Mozart & $1.657(0.024)$ & $1.721(0.015)$ & $1.684(0.005)$ & $1.692(0.024)$ & $1.724(0.019)$ & $\mathbf{1 . 7 0 6 ( 0 . 0 1 9 )}$ & $1.730(0.013)$ \\
Beethoven & $1.633(0.041)$ & $1.724(0.016)$ & $1.685(0.004)$ & $\mathbf{1 . 6 8 3 ( 0 . 0 2 4 )}$ & $1.712(0.020)$ & $1.709(0.023)$ & $1.722(0.023)$ \\
Jazz & $1.583(0.031)$ & $1.723(0.015)$ & $1.685(0.005)$ & $1.698(0.021)$ & $1.719(0.018)$ & $\mathbf{1 . 6 9 9 ( 0 . 0 1 9 )} 1.724(0.024)$ \\
\hline
\end{tabular}

LLE and FD distributions for the artificial piece do not differentiate for different composers. Consulting the distributions of compositions for all composers per map, in Figs. 5(d) and 5(f), one may notice that the LLE and FD distributions for the artificial pieces are vastly different for each map. Additionally, the fitness distributions per map, as shown in Fig. 5(b), seem to follow the behavior of the LLE distributions per map illustrated in Fig. 5(d). This observation is evaluated by the strong correlation (0.83) of the fitness and LLE values of the best individuals. This fact indicates that better fitness is expected to emerge from maps with positive LLE value closer to zero. Therefore, someone could arguably notice that there might be a threshold of "musical chaos" above but near zero LLE values. Such an argument however, needs more extensive scrutinization.

The mean values and standard deviations of the LLE and FD distributions for genuine and artificial compositions for each composer and map are shown in Tables 7 and 8 respectively. The LLEs of the genuine piece are exhibited to be closer to zero than any other LLE value of the artificial pieces in Table 7. The LLEs that correspond to the best mean fitness, as demonstrated in Table 5, are the smallest among all other artificial pieces, except from the piece of Beethoven. Similarly, the FD values of the genuine pieces is considerably lower than the FDs of the artificially composed pieces. In this case, the artificial pieces with the best fitness per composer are not the ones with the smallest FD value. It is therefore indicated, that there is no immediate connection between the FD value, at least as expressed by the computation of the time series algorithm, and the compositional capabilities of the dynamical systems.

\section{Synopsis and Concluding Remarks}

In this paper we presented a thorough study on the tonal composition potentiality of several discrete dynamical systems. Several genuine music masterpieces composed by J. S. Bach, Mozart, Beethoven and various jazz musicians, were utilized as music reference, providing compositional "guidelines" to the dynamical systems. Through an evolutionary approach, the parameters of the discussed systems were optimized so that the tonal sequences that each system composes, resemble the ones of the genuine pieces, according to some tonal features which encompass essential musical information. Firstly, a time series analysis was performed on the tonal sequences provided by the genuine composition that allowed an approximate phase space reconstruction of their attractor. Consequently, their Largest Lyapunov Exponent (LLE) and Fractal Dimension (FD) could be assessed, allowing a comparative analysis of their dynamical characteristics and the respective characteristics of the artificial pieces. The resulting artificial compositions were 
scrutinized in several aspects, which incorporated their tonal capabilities and dynamical characteristics for each composer and according to each tonal feature. Thereby, a thorough survey on the tonal compositional capabilities of each map was allowed, together with several comparative studies that provide insights about the qualitative music characteristics that may be pursued by utilizing dynamical systems as automatic music composers.

Through the time series analysis performed on the genuine musical masterpieces, an interesting finding is that the attractor characteristics of each composer differ significantly. The dynamical characteristics of these attractors however, could not be accurately reproduced by the examined dynamical systems. This is probably due to the fact that the examined dynamical systems are quite "simple" in terms of their dimensionality and time delay, as demonstrated by the respective phase space reconstruction values. However, some important insights were provided about the connection of qualitative music features and dynamical characteristics through the LLE value. Specifically, it was indicated that dynamical systems with smaller positive LLEs produced compositions which were more similar to the genuine pieces. Additionally, it may be assumed that iterative maps which performed better were the ones that could reproduce tonal motif-like structure, which is expressed through the features that incorporated percentages of ascending, descending or constant pitch intervals within short segments of music. Contrarily, the worst performing maps, were better at capturing less refined musical features, like absolute pitch differences and pitch-change gradient.

The fact that the reconstructed phase space of the original compositions incorporated a great number of dimensions, indicates that the appropriate dynamical systems should probably also incorporate more dimensions. Thereby, the task at hand could be approached by a similar evolutionary strategy, with iterative dynamical systems of higher dimensionality. The equations that constitute these systems however could incorporate a large number of parameters. For example, the general quadratic map, which constitutes a general form of a two-dimensional quadratic system incorporates 12 parameters, considering also the initial iterations. The $n$-dimensional version of the general quadratic map, for example, incorporates $2 n+2 n^{2}$ parameters and therefore their number becomes overwhelming even for a relatively small number of dimensions.

Future work could also include a similar time series analysis on pieces with more simple tonal structure, like dances or even contemporary popular songs. Thereby, the connections between the perceived tonal complexity and the dynamical characteristics, e.g. the fractal dimension, could be directly examined. Finally, future work should also incorporate the examination of the compositional capabilities of several dynamical systems under "special" parameter value combinations, which provoke "special" dynamical behavior. For instance, the compositional characteristics of the logistic function could be examined when its parameter is set at the Feigenbaum point, provoking "weakly chaotic" dynamics, through a similar comparison with genuine human compositions.

\section{Acknowledgments}

We gratefully acknowledge many stimulating and useful discussions with Professor Tassos C. Bountis. This research has been partially co-financed by the European Union [European Social Fund (ESF)] and Greek national funds through the Operational Program "Education and Lifelong Learning" of the National Strategic Reference Framework (NSRF) - Research Funding Program: Thales. Investing in knowledge society through the European Social Fund.

\section{References}

Ahmadi, B. \& Amirfattahi, R. [2010] "Comparison of correlation dimension and fractal dimension in estimating BIS index," Wireless Sensor Network 2, $67-73$.

Alevizos, P. D. \& Vrahatis, M. N. [2010] "Optimal dynamic box-counting algorithms," Int. J. Bifurcation and Chaos 20, 4067-4077.

Amador, A. \& Mindlin, G. B. [2008] "Beyond harmonic sounds in a simple model for birdsong production," Chaos 18, 043123-1-6.

Behrman, A. [1999] "Global and local dimensions of vocal dynamics," J. Acoust. Soc. America 105, 432443.

Bidlack, R. [1992] "Chaotic systems as simple (but complex) compositional algorithms," Comput. Music J. 16, 33-47.

Bilotta, E., Pantano, P., Cupellini, E. \& Rizzuti, C. [2007] "Evolutionary methods for melodic sequences 
generation from non-linear dynamic systems," Applications of Evolutionary Computing, Lecture Notes in Computer Science, Vol. 4448 (Springer, Berlin, Heidelberg), pp. 585-592.

Boon, J. P. \& Decroly, O. [1995] "Dynamical systems theory for music dynamics," Chaos 5, 501-508.

Chialvo, D. R. [2003] "How we hear what is not there: A neural mechanism for the missing fundamental illusion," Chaos 13, 1226-1230.

Choi, I. [1994] "Interactive exploration of a chaotic oscillator for generating musical signals in real-time concert performance," J. Franklin Instit. 331, 785-818.

Chua, L., Wu, C., Huang, A. \& Zhong, G.-Q. [1993] "A universal circuit for studying and generating chaos. i. Routes to chaos," IEEE Trans. Circuits Syst.-I: Fund. Th. Appl. 40, 732-744.

Coca, A. E., Tost, G. O. \& Zhao, L. [2010] "Characterizing chaotic melodies in automatic music composition," Chaos 20, 033125-1-12.

Dabby, D. S. [1996] "Musical variations from a chaotic mapping," Chaos 6, 95-107.

Esteller, R., Vachtsevanos, G., Echauz, J. \& Litt, B. [2001] "A comparison of waveform fractal dimension algorithms," IEEE Trans. Circuits Syst.-I: Fund. Th. Appl. 48, 177-183.

Fletcher, N. H. [1994] "Nonlinear dynamics and chaos in musical instruments," Compl. Int. 1, 106-117.

Fraser, A. \& Swinney, H. [1986] "Independent coordinates for strange attractors from mutual information," Phys. Rev. A 33, 1134-1140.

Goh, C., Hamadicharef, B., Henderson, G. T. \& Ifeachor, E. C. [2005] "Comparison of fractal dimension algorithms for the computation of EEG biomarkers for dementia," Proc. 2nd Int. Conf. Computational Intelligence in Medicine and Healthcare (CIMED2005) (Lisbon, Portugal), pp. 464-471.

Grassberger, P. \& Procaccia, I. [1983] "Measuring the strangeness of strange attractors," Physica D 9, 189208.

Harley, J. [1994] "Algorithms adapted from chaos theory: Compositional considerations," Proc. 1993 Int. Computer Music Conf. (ICMA 1994) (San Francisco, USA), pp. 209-212.

Herman, M. [1993] "Deterministic chaos, iterative models, dynamical systems and their application in algorithmic composition," Proc. 1993 Int. Computer Music Conf. (ICMA 1993) (San Francisco, USA), pp. 194-197.

Higuchi, T. [1988] "Approach to an irregular time series on the basis of the fractal theory," Physica D 31, 277283.

Jafari, A., Almasganj, F. \& Bidhendi, M. N. [2010] "Statistical modeling of speech Poincaré sections in combination of frequency analysis to improve speech recognition performance," Chaos 20, 033106-1-11.
Kaliakatsos-Papakostas, M. A., Floros, A. \& Vrahatis, M. N. [2012a] "Intelligent real-time music accompaniment for constraint-free improvisation," Proc. 24th IEEE Int. Conf. Tools with Artificial Intelligence (ICTAI 2012) (Piraeus, Athens, Greece), pp. 444-451.

Kaliakatsos-Papakostas, M. A., Floros, A. \& Vrahatis, M. N. [2012b] "Music synthesis based on nonlinear dynamics," Proc. Bridges 2012, Mathematics, Music, Art, Architecture, Culture (Baltimore, USA), pp. 467470 .

Kaliakatsos-Papakostas, M. A., Floros, A. \& Vrahatis, M. N. [2013] "Evodrummer: Deriving rhythmic patterns through interactive genetic algorithms," Evolutionary and Biologically Inspired Music, Sound, Art and Design (Springer, Berlin, Heidelberg), pp. 25-36.

Kantz, M. J. [1988] "Fractals and the analysis of waveforms," Comput. Biol. Med. 18, 145-156.

Kennel, M. B., Brown, R. \& Abarbanel, H. D. I. [1992] "Determining embedding dimension for phasespace reconstruction using a geometrical construction," Phys. Rev. A 45, 3403-3411.

Kodba, S., Perc, M. \& Marhl, M. [2005] "Detecting chaos from a time series," Eur. J. Phys. 26, 205-215.

Kugiumtzis, D. \& Tsimpiris, A. [2010] "Measures of analysis of time series (mats): A matlab toolkit for computation of multiple measures on time series data bases," J. Statist. Softw. 33, 1-30.

Lai, Y.-C. \& Ye, N. [2003] "Recent developments in chaotic time series analysis," Int. J. Bifurcation and Chaos 13, 1383-1422.

Manaris, B., Romero, J., Machado, P., Krehbiel, D., Hirzel, T., Pharr, W. \& Davis, R. B. [2005] "Zipf's law, music classification, and aesthetics," Computer Music J. 29, 55-69.

Mandelbrot, B. B. [1982] The Fractal Geometry of Nature (W. H. Freeman and Company).

Oudre, L., Févotte, C. \& Grenier, Y. [2010] "Probabilistic framework for template-based chord recognition," 2010 IEEE Int. Workshop on Multimedia Signal Processing (MMSP), (Saint-Malo, France), pp. 183-187.

Petrosian, A. [1995] "Kolmogorov complexity of finite sequences and recognition of different preictal EEG patterns," Proc. Eighth Ann. IEEE Symp. ComputerBased Medical Systems, CBMS '95 (IEEE Computer Society, Washington, DC, USA), pp. 212-217.

Polychronaki, G. E., Ktonas, P. Y., Gatzonis, S., Siatouni, A., Asvestas, P. A., Tsekou, H., Sakas, D. \& Nikita, K. S. [2010] "Comparison of fractal dimension estimation algorithms for epileptic seizure onset detection," J. Neural Engin. 7, 046007.

Pressing, J. [1988] "Nonlinear maps as generators of musical design," Comput. Music J. 12, 35-46.

Price, K., Storn, R. M. \& Lampinen, J. A. [2005] Differential Evolution: A Practical Approach to Global 
Optimization (Natural Computing Series) (SpringerVerlag, NY, Inc., Secaucus, NJ, USA).

Raghavendra, B. S. \& Dutt, N. D. [2010] "Computing fractal dimension of signals using multiresolution box-counting method," Int. J. Inform. Math. Sci. 1, 50-65.

Rizzuti, C., Bilotta, E. \& Pantano, P. [2009] "A GA-based control strategy to create music with a chaotic system," Applications of Evolutionary Computing, Lecture Notes in Computer Science, Vol. 5484 (Springer, Berlin, Heidelberg), pp. 585-590.

Rosenstein, M. T., Collins, J. J. \& De Luca, C. J. [1993] "A practical method for calculating largest Lyapunov exponents from small data sets," Physica D 65, 117134.

Sevcik, C. [1998] "A procedure to estimate the fractal dimension of waveforms," Compl. Int. 5. Available from http://www.complexity.org.au/ci/vol05/sevcik/ sevcik.html.

Shannon, C. E. [2001] "A mathematical theory of communication," ACM SIGMOBILE Mobile Computing and Commun. Rev. 5, 3-55.
Storn, R. \& Price, K. [1997] "Differential evolution — A simple and efficient adaptive scheme for global optimization over continuous spaces," J. Global Optim. 11, 341-359.

Takens, F. [1981] "Detecting strange attractors in turbulence," Dynamical Systems and Turbulence, Warwick 1980 (Springer, Berlin, Heidelberg), pp. 366-381.

Tokuda, I., Riede, T., Neubauer, J., Owren, M. J. \& Herzel, H. [2002] "Nonlinear analysis of irregular animal vocalizations," J. Acoust. Soc. America 111, 2908-2919.

Tricot, C. [1994] Curves and Fractal Dimension (Springer).

Voss, R. F. \& Clarke, J. [1978] "1/f noise in music: Music from 1/f noise," J. Acoust. Soc. America 63, 258-263.

Wilcoxon, F. [1945] "Individual comparisons by ranking methods," Biometr. Bull. 1, 80-83.

Wolf, A., Swift, J. B., Swinney, H. L. \& Vastano, J. A. [1985] "Determining Lyapunov exponents from a time series," Physica D 16, 285-317. 